\title{
3D heat flux effects in the experimental evaluation of corrosion by IR thermography
}

\author{
by S. Marinetti, P.G. Bison and E. Grinzato
}

National Research Council - Institute of Building Technology, Section of Padova, Corso

Stati Uniti 4, 35127 Padova, ITALY, e-mail: sergio.marinetti@itef.pd.cnr.it

\begin{abstract}
IR thermography has been successfully applied to corrosion detection in metals. The main advantage of such a technique is its capability to inspect large surfaces in a short time. A simple formula for corrosion evaluation was proposed in 1996. It is based on the assumption of one-dimensional heat diffusion and was successfully applied to estimate the material loss for large defects. In this paper the case of small sized defects is analysed. When the 3D heat diffusion effects are strong, the formula yields underestimated values. Results of numerical simulations are used to devise a method which makes the estimate of the material loss more accurate.
\end{abstract}

\section{Introduction}

Thermal Non-Destructive Evaluation (TNDE) of corrosion in metals has proved to be a reliable and fast non-contact method [1,2]. The acoustic and eddy current NDT remain the most used methods due to their sensitivity. Nevertheless their use encounters difficulties when the surface to be tested is large and the inspection time is limited.

The ever growing performances of IR imagers, image processing, thermal models, and heat sources make IR thermography more and more interesting from a quantitative point of view. To exploit the main feature of thermal tests, i.e. the capability of inspecting large areas in a short time, fast processing algorithms are needed.

A robust and simple inversion algorithm to evaluate IR images in terms of material loss was proposed for large corroded areas [3]. It is based on the assumption that the heat flux through the corroded and sound regions is one-dimensional. This requirement is not met when the defect extension is small. In such a case the material loss is underestimated. In the present work, corrosion is simulated by a commercial FEM code in order to better understand the role played by the main variables involved in the thermal process. As a result, a compensation curve is applied to the underestimates to improve the accuracy of the material loss evaluation.

A steel specimen of $3 \mathrm{~mm}$ in thickness is experimentally tested and results are reported.

\section{1D case: theoretical model and inversion formula}

In the field of TNDE, corrosion is generally treated as a reduction of the wall thickness. Possible changes in material properties due to chemical reactions are neglected. The sample considered here is a slab. As to the defect shape, the most common numerical models deal with disk-shapes in 2D cylindrical coordinates and channel-like shapes in 2D Cartesian space. Large defects, where the thermal process occurs independently with respect to the sound area, are modelled by analytical solutions of the heat diffusion equation [4].

The amount of corrosion is expressed as the relative material loss: $\lambda=\left(L_{s}-L_{d}\right) / L_{s}$ where $L_{s}$ and $L_{d}$ are the thickness of the sound and corroded areas, respectively.

Let us consider a 1D model for a slab of thickness $L$ heated by a Dirac heat pulse (i.e. pulse duration is negligible). For thermally high conductive material and small thickness, 
the process could be considered as adiabatic. The temperature evolution of the heated surface (front surface) is governed by the following equation where $\alpha$ is thermal diffusivity, $K$ thermal conductivity, $W$ pulse energy density, $\tau$ time and Fo the Fourier number.

$$
T(\tau, L)=\frac{W \alpha}{K L}\left(1+2 \sum_{n=1}^{+\infty} e^{-n^{2} \pi^{2} F_{0}}\right) \quad \text { Fo }=\frac{\alpha \tau}{L^{2}}
$$

The asymptotic temperature value, corresponding to the end of the process $(\tau \rightarrow \infty)$ is $T(\tau \rightarrow \infty, L)=(W \alpha) /(K L)$. From the knowledge of the asymptotic temperatures over the defect $T_{d}\left(\tau \rightarrow \infty, L_{d}\right)$ and the sound area $T_{s}\left(\tau \rightarrow \infty, L_{s}\right)$ it is possible to recover the material loss $\lambda$ through the following simple formula [3]:

$$
\lambda=1-\frac{T_{s}\left(\tau \rightarrow \infty, L_{s}\right)}{T_{d}\left(\tau \rightarrow \infty, L_{d}\right)}
$$

In practice, when defects cannot be considered as infinitely extended, 3D heat diffusion effects become important. The asymptotic temperature over the defect is affected by the heat exchanged with the surrounding sound material. As a result, eq. (2) provides an underestimate of $\lambda$. Another aspect to be taken into account is the sensitivity of the front surface temperature to the sample thickness. This parameter is very low at the beginning and maximum at the end of the process. It has been shown [3] that it is convenient to apply eq. (2) at the observation time $F_{O}=0.68$. Indeed, at that time, the $3 \mathrm{D}$ heat effects are still weak and the sensitivity to the rear surface is already high enough.

\section{2D case: numerical simulation}

In order to determine the limits of the 1D approach, a set of numerical simulations was performed using a commercial FEM package (FEMLAB ${ }^{\circledR}$ by COMSOL AB) implemented in the MATLAB ${ }^{\circledR}$ environment. The model adopted is $2 D$ axisymmetrical (Figure 1a). The heating pulse was modelled as a Gaussian time function with a standard deviation of 1 ms. Moreover the surface heat exchange coefficient $h$ was set to $10 \mathrm{Wm}^{-2} \mathrm{~K}^{-1}$ in order to make simulations closer to the actual experimental conditions. Nevertheless, dealing with metals and therefore with low Biot numbers, the results reported below will not differ much from those that we would obtain in adiabatic conditions. Thermal conductivity and diffusivity were set to $62 \mathrm{Wm}^{-1} \mathrm{~K}^{-1}$ and $1.6510^{-5} \mathrm{~m}^{2} \mathrm{~s}^{-1}$ (steel), respectively. The material loss $\lambda$ varied from $20 \%$ to $50 \%$. A first set of simulations was performed on a $3 \mathrm{~mm}$ thick sample and considering defects with diameter $D_{d}$ ranging from 2 to $24 \mathrm{~mm}$. At each run the front surface temperature over the centre of the defect and the sound area was considered and eq. (2) applied at Fo $=0.68$. In Table 1 corrosion estimates $\lambda_{\text {est }}$ are reported for $\lambda=0.2,0.3$, and 0.5 . As expected, the underestimate increases as the defect diameter decreases.

Table 1: Corrosion estimates from numerical simulations (steel): $3 \mathrm{~mm}$ thick sample with defect diameter ranging from $2 \mathrm{~mm}$ to $24 \mathrm{~mm}$.

\begin{tabular}{|l|rrrrrrr|}
\hline$D_{d}[\mathrm{~mm}]$ & 2.0 & 5.0 & 7.5 & 10.0 & 15.0 & 20.0 & 24.0 \\
\hline$\lambda_{\text {est }}$ at $\mathrm{Fo}=0.68(\lambda=0.2)$ & 0.010 & 0.059 & 0.109 & 0.150 & 0.188 & 0.197 & 0.198 \\
\hline$\lambda_{\text {est }}$ at $\mathrm{Fo}=0.68(\lambda=0.3)$ & 0.016 & 0.091 & 0.170 & 0.229 & 0.285 & 0.297 & 0.298 \\
\hline$\lambda_{\text {est }}$ at $\mathrm{Fo}=0.68(\lambda=0.5)$ & 0.026 & 0.163 & 0.304 & 0.400 & 0.479 & 0.496 & 0.498 \\
\hline
\end{tabular}

Figure 2 shows the data of Table 1 after interpolation by spline functions. It can be noticed that diameters larger than $20 \mathrm{~mm}$ ensure that the assumption of $1 \mathrm{D}$ heat flux is 
met. In such a case, eq. (2) provides a good corrosion evaluation ( $99 \%$ of the true value). To investigate the influence of geometrical properties, a second set of simulations was performed for a sample made of the same material as before, but with a double thickness $(L=6 \mathrm{~mm})$, defect diameter $D_{d}$ varying from $4 \mathrm{~mm}$ to $48 \mathrm{~mm}$ and $\lambda=0.5$. Results are reported in Table 2.

Table 2: Corrosion estimates from numerical simulations (steel): $6 \mathrm{~mm}$ thick sample with defect diameter ranging from $4 \mathrm{~mm}$ to $48 \mathrm{~mm}$.

\begin{tabular}{|l|rrrrrrr|}
\hline$D_{d}[\mathrm{~mm}]$ & 4.0 & 10.0 & 15.0 & 20.0 & 30.0 & 40.0 & 48.0 \\
\hline$\lambda_{\text {est }}$ at Fo=0.68 $(\lambda=0.5)$ & 0.026 & 0.163 & 0.305 & 0.400 & 0.479 & 0.496 & 0.498 \\
\hline
\end{tabular}

In this case, the 1D heat flux is ensured with diameters exceeding $40 \mathrm{~mm}$. In Figure 3 data from Table 2 and the corresponding row of Table 1 are plotted.

Finally to understand the role played by the thermal properties, another set of simulations was performed considering a constant geometry: $6 \mathrm{~mm}$ thick sample with a defect diameter $D_{d}$ of $10 \mathrm{~mm}$ and $\lambda=0.2$. A second material with $K=0.64 \mathrm{Wm}^{-1} \mathrm{~K}^{-1}$ and $\alpha=5.210^{-7} \mathrm{~m}^{2} \mathrm{~s}^{-1}$ (CFRP) was introduced. Keeping the same value of $h\left(10 \mathrm{Wm}^{-2} \mathrm{~K}^{-1}\right)$ and with the thermal conductivity of CFRP 100 times smaller than that of steel, the Biot number is 100 times larger. This makes surface heat exchange not negligible. Hence, for a more complete analysis, both values $h=10 \mathrm{Wm}^{-2} \mathrm{~K}^{-1}$ and $h=0$ were considered for CFRP. In Table 3 the results are reported.

Table 3: Corrosion estimates from numerical simulations: $6 \mathrm{~mm}$ thick sample with a defect diameter of $10 \mathrm{~mm}$ and $\lambda=0.2$. Two different materials are considered: steel and CFRP.

\begin{tabular}{|c|ccc|}
\cline { 2 - 4 } \multicolumn{1}{c|}{} & $D_{d}[\mathrm{~mm}]$ & $h\left[\mathrm{Wm}^{-2} \mathrm{~K}^{-1}\right]$ & $\lambda_{\text {est }}$ at Fo=0.68 $(\lambda=0.2)$ \\
\hline Steel & 10 & 10 & 0.059 \\
\hline CFRP & 10 & 10 & 0.045 \\
\hline CFRP & 10 & 0 & 0.059 \\
\hline
\end{tabular}

Results show that two samples with the same geometry but made of different materials exhibit the same corrosion underestimate if the surface heat exchange is negligible (first and last row of Table 3).

\section{Compensation curve}

The results obtained in the previous section could already provide a kind of abacus to predict the error made by using eq. (2). Indeed, once the defect diameter $D_{d}$ and the corrosion estimate $\lambda_{\text {est }}$ are known, from an abacus like the one shown in Figure 2 , it is possible to recover the correct value of $\lambda$. Such a value is the asymptotic value of the curve passing through the point $\left(D_{d}, \lambda_{\text {est }}\right)$. The main drawback of this procedure is the need to compute as many curves as the levels of corrosion of interest. To overcome this problem, let us consider the curves of Figure 2 normalized by their asymptotic values (Figure 4). It can be seen that all the curves could be well approximated by their average profile (solid line).

Now we have one single curve for all corrosion levels between $20 \%$ and $50 \%$, but this curve has been computed for a given thickness $(L=3 \mathrm{~mm})$. Figure 3 shows how the same defect diameter causes two different estimates depending on the sample thickness. To put these curves together (Figure 3 ), we considered the ratio $D_{d} / L$ (Figure 5). Finally in Figure 
6 the resulting compensation curve is shown. It provides the forecast of underestimate for a given ratio $D_{d} / L$. This compensation was applied to the values reported in the Tables above with the results shown in Figure 7.

\section{Experimental results}

A steel specimen with $3 \mathrm{~mm}$ thickness has been experimentally tested to verify the performance of the compensation procedure when applied to experimental data. As shown in Figure 1b, the sample has 6 circular flat bottom holes with a diameter of $10 \mathrm{~mm}$ located at different depths corresponding to $50 \%, 30 \%, 20 \%, 10 \%, 5 \%$ and $2 \%$ of material loss. Only the first three defects were considered in the following analysis.

The sample was heated by two flash tubes delivering $4800 \mathrm{~J}$ pulse energy in $5 \mathrm{~ms}$. The cooling process of the heated surface was observed for $2 \mathrm{~s}$ with an IR QWIP camera (ThermaCam $^{\mathrm{TM}}$ SC3000 from FLIR Sytems) operating in the spectral range from 8 to 9 $\mu \mathrm{m}$. Two thermogram sequences were acquired at frame rates of $50 \mathrm{~Hz}(240 \times 320$ pixel per frame) and $150 \mathrm{~Hz}(80 \times 320$ pixels per frame).

\subsection{Thermal diffusivity measurement}

The use of the compensation curve requires the knowledge of the defect diameter, the sample thickness and the first material loss estimate provided by eq. (2). As mentioned above, it was found that a convenient observation time for eq. (2) is Fo=0.68. To convert this dimensionless parameter into time, the thermal diffusivity must be known. Its value could be taken from literature, if available, or estimated experimentally. Among the many IR methods for diffusivity measurements [5], the choice went to the one that was thought to be of practical interest. It is based on eq. (1) reported below in a dimensionless form:

$$
\frac{T(F o)}{T(F O \rightarrow \infty)}=1+2 \sum_{n=1}^{+\infty} e^{-n^{2} \pi^{2} F o}
$$

The quantity $T(F o) \cdot F^{1 / 3}$ (where $T(F o$ ) is the front surface temperature evolution after the flash heating) exhibits a local minimum at $F_{O}=\left(\alpha \cdot t_{\min }\right) / L^{2}=0.2656$. Therefore, plotting $T(t) \cdot t^{1 / 3}$ vs. $t$ (where $t$ is time), the diffusivity is linked to the time of minimum $t_{\min }$ through

$$
\alpha=\frac{0.2656 \cdot L^{2}}{t_{\min }}
$$

The experimental data used to this purpose were those acquired at $150 \mathrm{~Hz}$ because of the higher resolution in time. The result of eq. (4) was $\alpha=1.6510^{-5} \mathrm{~m}^{2} \mathrm{~s}^{-1}$.

\subsection{Defect diameter measurement}

The other quantity that must be known is the defect diameter. This information can be extracted from the thermogram sequence.

The main drawback of defect sizing by thermal techniques is that the hot spot is seldom a perfect representation of the true defect shape. A detailed analysis of the defect sizing methods is beyond the purpose of this paper $[6,7,8]$.

The followed procedure was used to determine the diameter of defects by considering a spatial profile across the defect taken at the time of maximum visibility (Figure 8 ) [9]. The apparent size was then defined as the locus at half maximum $\Delta T$ (i.e. the difference between the surface temperature of each point and a reference area). To convert the size expressed in pixel into $\mathrm{mm}$, a calibrated object has been put in the field of view of the camera. Measurements of defect size are reported in Table 4. 


\subsection{Corrosion evaluation}

Eq. (2) was applied at a time delay of $0.37 \mathrm{~s}$ after the flash $(\mathrm{Fo}=0.68)$ and provided the first estimates $\lambda_{\text {est }}$. For each defect the ratio $D_{d} / L$ was computed and the compensation factor C.F. was recovered from Figure 6. Finally, dividing $\lambda_{\text {est }}$ by C.F. we obtained the corrected corrosion estimates. The results are reported in Table 4.

Table 4: Experimental results (sample thickness: $L=3 \mathrm{~mm}$ ).

\begin{tabular}{|c|c|c|c|c|c|c|c|}
\hline$\lambda_{\text {true }}$ & $\begin{array}{c}\text { Apparent } \\
\text { size }[\mathrm{mm}]\end{array}$ & $D_{d} / L$ & $\lambda_{\text {est }}$ at Fo=0.68 & $\begin{array}{c}\lambda_{\text {est }} \\
\text { error [\%] }\end{array}$ & C.F. & $\lambda_{\text {est }} / C . F$. & $\begin{array}{c}\lambda_{\text {est }} / C . F . \\
\text { error [\%] }\end{array}$ \\
\hline 0.20 & 9.43 & 3.14 & 0.117 & -41.5 & 0.72 & 0.163 & -18.5 \\
0.30 & 8.73 & 2.91 & 0.196 & -34.7 & 0.67 & 0.294 & -2.0 \\
0.50 & 8.73 & 2.91 & 0.335 & -33.0 & 0.67 & 0.502 & 0.4 \\
\hline
\end{tabular}

\section{Conclusions}

This paper contains the results of a numerical analysis for 2D models of corrosion. Efforts have been made to present results and graphs in a dimensionless form allowing the extension of conclusions to a wide range of sample geometries and to any material. Starting from a formula for corrosion evaluation derived from a 1D model, the analysis presented here tries to extend its use to the case of $3 \mathrm{D}$ heat diffusion. As a final result, a compensation curve providing the correction factor is devised. Verification of compensation procedure has been made with numerical $2 \mathrm{D}$ models: errors were up to $90 \%$ before compensation and less than $10 \%$ thereafter. A steel sample with circular flat bottom holes has been tested. Experimental results showed an appreciable increase of the accuracy.

\section{REFERENCES}

[1] ALCOTT, J.: "An investigation of nondestructive inspection equipment: detecting hidden corrosion on USAF aircraft". Material Evaluations, 52(1), January 1994, p 64-73.

[2] SYED, H. I. and CRAMER, K. E.: "Corrosion detection in aircraft skin". Proceedings SPIE Vol. 1933: Thermosense XV, Orlando (Florida), 14-16 April 1993, p 160-163.

[3] VAVILOV, V.; GRINZATO, E.; BISON, P. G.; MARINETTI, S. and BALES, M. J.: "Surface transient inversion for hidden corrosion characterisation: theory and applications". Int. J. Heat Mass Transfer, Vol. 39 No. 2, 1996, p 355-371.

[4] CARSLAW, H. S.; JAEGER, J. C.: "Conduction of heat in solids". Oxford University Press, London, 1959.

[5] CERNUSCHI, F.; FIGARI, A.; LORENZONI, L.; BISON, P. G.; MARINETTI, S. and GRINZATO, E.: "Comparison of thermal diffusivity measurement techniques". Proceedings of the $6^{\text {th }}$ International QIRT Conference, Dubrovnik (Croatia), 24-27 September 2002, to be published.

[6] LAU, K. S. and ALMOND, D. P.: "Defect sizing by transient thermography I: An analytical treatment". J. Phys. D: Appl. Phys, 27, p 1063-1069.

[7] SAINTEY, M. B. and ALMOND, D. P.: "Defect sizing by transient thermography II: a numerical treatment". J. Phys. D: Appl. Phys, 28, p 2539-2546.

[8] CERNUSCHI, F.; LUDWIG, N. and TERUZZI, P.: "Statistics-based procedure for defect sizing and experimental evaluation of convection phenomena by using Video Pulsed Thermography". Proceedings of the $4^{\text {th }}$ International QIRT Conference, Lodz (Poland), 7-10 September 1998, p 30-35.

[9] KRAPEZ, J. C.: "Thermal contrast in pulsed infrared thermography". Nondestructive Testing Handbook: Infrared and Thermal Testing, Vol. 3, chapter 13, ASNT, 2001,p 411-439. 


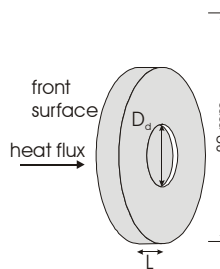

a)

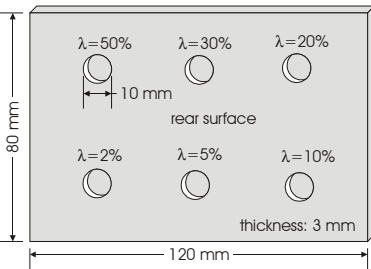

b)

Fig. 1. Model used for $2 D$ simulations (a), sketch of the experimentally tested sample (b)

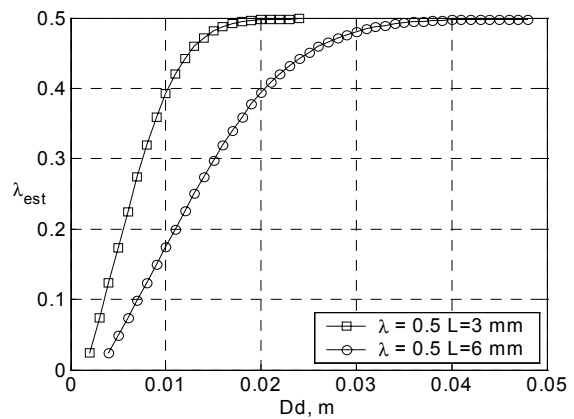

Fig. 3. $\lambda_{\text {est }}$ vs. $D_{d}$ for $\lambda=0.5$ and different thickness ( $3 \mathrm{~mm}$ and $6 \mathrm{~mm}$ )

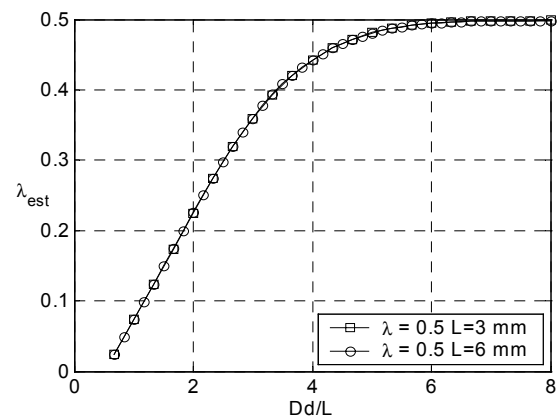

Fig. 5. $\lambda_{\text {est }} v s . D_{d} / L$ for $\lambda=0.5$ and different thickness ( $3 \mathrm{~mm}$ and $6 \mathrm{~mm}$ )

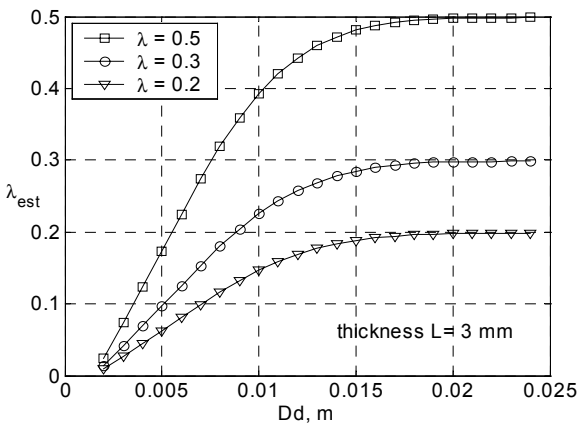

Fig. 2. Results of eq. (2) $\lambda_{\text {est }}$ vs. defect diameter $D_{d}(L=3 \mathrm{~mm})$

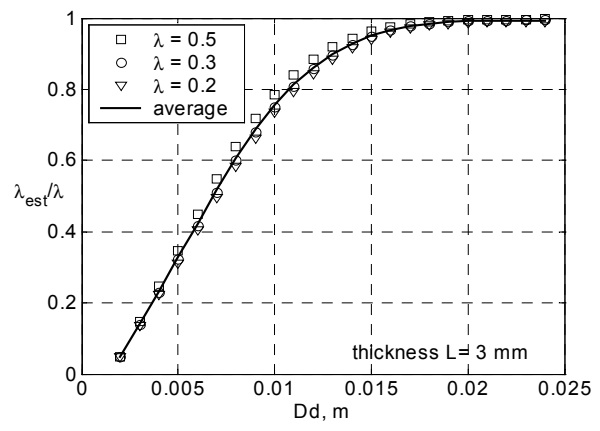

Fig. 4. Results of eq. (2) normalized by the true corrosion value vs. $D_{d}$

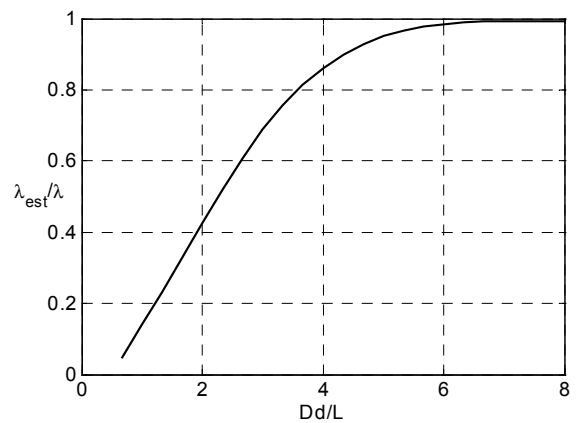

Fig. 6. Compensation curve $\lambda_{\mathrm{est}} / \lambda$ vs. $D_{d} / L$ 


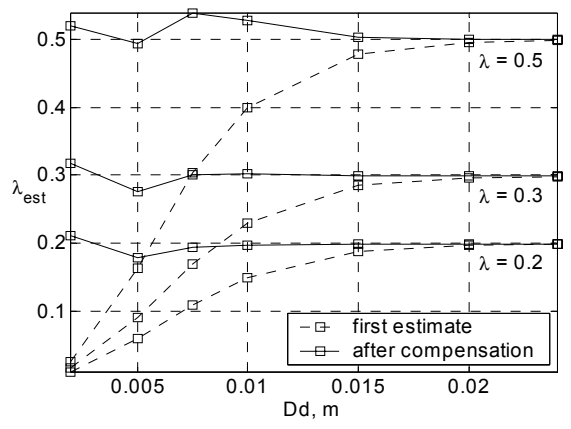

Fig. 7. Numerical results of the inversion procedure

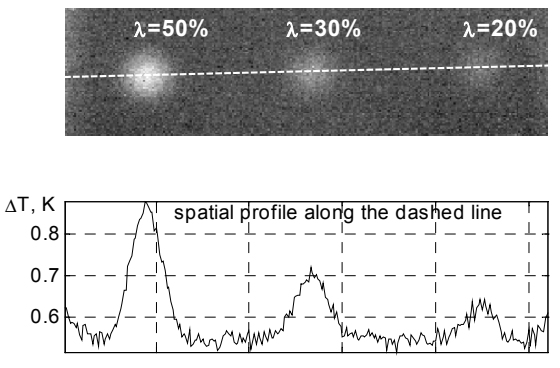

Fig. 8. Raw thermogram and spatial profile at $t=0.28 \mathrm{~s}$ 